\title{
The Biomechanical Role of Scaffolds in Augmented Rotator Cuff Tendon Repairs
}

Amit Aurora

Cleveland Clinic

Jesse A. McCarron

Department of VA Medical Center

Antonie J. van den Bogert

Cleveland State University, a.vandenbogert@csuohio.edu

POIIOW FiGati\&additional works at: https://engagedscholarship.csuohio.edu/enme_facpub

I'nveland State University, j.gatica@csuohio.edu

Part of the Biomechanics and Biotransport Commons

Hespelbe $P_{s}$ lapeests to this work benefit you? Let us know!

Gever/hshelipis Statement

NOTICE: this is the author's version of a work that was accepted for publication in Journal of

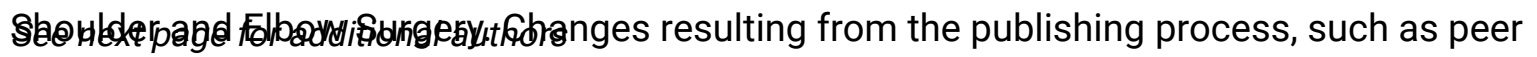
review, editing, corrections, structural formatting, and other quality control mechanisms may not be reflected in this document. Changes may have been made to this work since it was submitted for publication. A definitive version was subsequently published in Journal of Shoulder and Elbow Surgery, 21, 8, (08-01-2012); 10.1016/j.jse.2011.05.014

\section{Original Citation}

Aurora, A., McCarron, J. A., van den Bogert, A.J., Gatica, J. E., lannotti, J. P., \& Derwin, K. A. (2012). The biomechanical role of scaffolds in augmented rotator cuff tendon repairs. Journal of Shoulder and Elbow Surgery, 21(8), 1064-1071. doi: 10.1016/j.jse.2011.05.014

This Article is brought to you for free and open access by the Mechanical Engineering Department at EngagedScholarship@CSU. It has been accepted for inclusion in Mechanical Engineering Faculty Publications by an authorized administrator of EngagedScholarship@CSU. For more information, please contact library.es@csuohio.edu. 


\section{Authors}

Amit Aurora, Jesse A. McCarron, Antonie J. van den Bogert, Jorge E. Gatica, Joseph P. Iannotti, and Kathleen A. Derwin 


\title{
The biomechanical role of scaffolds in augmented rotator cuff tendon repairs
}

\author{
Amit Aurora, D Eng ${ }^{a, b}$, Jesse A. McCarron, MDc, Antonie J. van den Bogert, $\mathrm{PhD}^{\mathrm{d}}$, \\ Jorge E. Gatica, PhD ${ }^{b}$, Joseph P. Iannotti, MD, PhD ${ }^{\mathrm{e}}$, Kathleen A. Derwin, $\mathrm{PhD}^{\mathrm{a}, \mathrm{e}, *}$
}

${ }^{a}$ Department of Biomedical Engineering and Orthopedic Research Center, Cleveland Clinic, Cleveland, OH, USA

${ }^{b}$ Department of Chemical and Biomedical Engineering, Cleveland State University, Cleveland, OH, USA

${ }^{c}$ Orthopedic Surgery Section, Department of VA Medical Center, Portland, OR, USA

${ }^{d}$ Orchard Kinetics LLC, Cleveland Heights, Cleveland, OH, USA

${ }^{e}$ Department of Orthopedic Surgery, Orthopedic and Rheumatologic Institute, Cleveland Clinic, Cleveland, OH, USA

Investigational Review Board approval was not required for this study. *Reprint requests: Kathleen A. Derwin, $\mathrm{PhD}$, Department of Biomedical Engineering, ND2-20, Cleveland Clinic, 9500 Euclid Ave, Cleveland, OH 44122, USA

E-mail address: derwink@ccf.org (K.A. Derwin).
An estimated 75,000 rotator cuff surgeries are performed annually in the United States. ${ }^{24}$ Although surgical treatment and rehabilitation strategies continue to evolve, the surgical management of these tears still poses a significant challenge to the orthopedic community, as evidenced by the $20 \%$ to 
90\% repair failure rates reported after rotator cuff repair surgery. ${ }^{1,6,7,12-14,16}$ High surgical failure rates can be attributed to a number of biologic, anatomic, and mechanical factors, which include inferior tissue quality, tendon retraction, muscle atrophy and fatty infiltration, undue tension at the repair site in the early postoperative period, and the synovial fluid environment. ${ }^{5,8,15,22,23}$ Although different repair strategies and hardware, immobilization protocols, and postoperative rehabilitation approaches have been used to reduce rotator cuff repair failure rates, ${ }^{11,17-19,21}$ structural repair failure remains a problem. Hence, there is a critical need to develop mechanical and/or biologic augmentation repair strategies to increase the clinical success of these repairs.

During the last decade, natural and synthetic biomaterials have been developed as scaffolds for tendon repair augmentation. Currently, scaffolds derived from various natural and synthetic biomaterials are being marketed as augmentation devices for rotator cuff repairs at the time of surgery. ${ }^{3,9,10}$ The US Food and Drug Administration (FDA) has cleared these devices "to support soft tissues where weakness exists" but not "to provide the full mechanical strength for the tendon repair." The mechanical connotation of their intended use leads to the common belief that when applied appropriately, these devices may provide some degree of load-sharing of forces across the tendon repair site and thus decrease the likelihood of tendon retear.

Although significant advances have been made in the development of scaffolds for rotator cuff repair augmentation, there are limited experimental data to support the notion that scaffold augmentation of a tendon repair will actually improve the biomechanical performance of the repair construct. McCarron et $\mathrm{al}^{20}$ recently showed that augmentation with a polylactic acid scaffold device (X-Repair, Synthasome Inc, San Diego, CA, USA) significantly increased the yield load (56\%-92\%) and ultimate load (56\%-76\%) of rotator cuff repairs in a human cadaver model. ${ }^{20} \mathrm{X}$-Repair augmentation also altered the mode of repair failure: failure by sutures cutting through the tendon was reduced, occurring in 17 of 20 nonaugmented repairs but in only 7 of 20 augmented repairs. In another study, Barber et $\mathrm{al}^{4}$ demonstrated a $19 \%$ increase in failure load and fewer failures at the suture-tissue interface for supraspinatus repairs augmented with GraftJacket (Human Acellular Dermis, Wright Medical, Arlington, VA, USA) compared with nonaugmented repairs.

Although these studies demonstrate the potential for scaffold augmentation to improve the initial biomechanical properties of a rotator cuff repair construct, the appropriate scaffold material properties and/or surgical application techniques for achieving optimal biomechanical performance in the setting of rotator cuff repairs are unknown. Furthermore, no studies to date have investigated the percent load carried by a scaffold when used for rotator cuff repair augmentation.

To address these questions and enhance our understanding of the basic mechanics of scaffold augmentation, we recently developed and validated a spring-network model for nonaugmented and augmented human rotator cuff repairs. ${ }^{2}$ The objectives of the current study are now to use this model to predict (1) the manner in which simulated changes to components of the tendon repair, such as reduced tendon quality, altered surgical technique, and different scaffold designs, influence the biomechanical performance (yield load and stiffness) of the repair construct and (2) the percent load carried by the scaffold augmentation component of the repair construct in each of these simulated clinical scenarios.

\section{Materials and methods}

We previously developed and validated a spring-network model for simplified nonaugmented and augmented human rotator cuff repairs, based on the physics of springs in series and in parallel. ${ }^{2}$ For the cadaveric rotator cuff repairs, a strip of the superior infraspinatus tendon (12 mm wide) was released and repaired to the greater tuberosity using a double-row transosseous technique with 2 Mason Allen sutures per row. ${ }^{20}$ The prototypical augmentation graft used for the augmented repairs was a poly-L-lactic acid (PLLA) scaffold (X-Repair, Synthasome Inc, San Diego, CA, USA). The nonaugmented repair was modeled as 2 springs in series (Fig.1, $A$ and $B$ ), and the augmented repair was modeled as a combination of 5 springs in series and parallel (Fig. 1, $C$ and $D$ ). The individual spring components, representing the points of compliance of the repair constructs, were modeled as nonlinear springs (Table I). The springs representing the tendon (spring \#2), scaffold augmentation component (spring \#3) and scaffold-tendon attachment (spring \#4) were modeled using a single phase nonlinear equation, $F=F^{o}+A x^{b}$, and the spring representing the tendon-to-bone repair (spring \#1) was modeled using a biphasic nonlinear equation, $F=F^{o}+\frac{A x^{b}}{1+B x^{c}}$. In these equations, force $(F)$ is a function of the displacement $(x)$ of the individual spring component. The parameters $F^{o}, A, B, b$, and $c$ were estimated using nonlinear least-squares analysis of experimental data from each individual component. The aggregate spring-network models were validated by comparing the model predictions to in vitro experimental data in the failure-loading region of repair constructs that were preconditioned for 100 cycles from 50 to $150 \mathrm{~N}$ at $0.25 \mathrm{~Hz}$ and subsequently distracted to failure with uniaxial loading in tension at $30 \mathrm{~mm} / \mathrm{min}^{20}$ Further details of the model and its validation have previously been described. ${ }^{2}$

In the current study, the validated models were varied parametrically to simulate clinically relevant scenarios, namely, changes in tendon quality, altered surgical technique(s) and different scaffold designs. More specifically, parameter $A$ of the tendon-to-bone repair (spring \#1), the scaffold augmentation component (spring \#3), and the scaffold-tendon attachment (spring \#4) was varied from its respective baseline value, while keeping other parameters at their respective baseline values. (The baseline values are those derived from the actual experimental data). Although the parameter $A$ itself does not have any particular physical significance, it is a proportionality constant associated with changes in load-displacement characteristics of a given spring component and hence can be varied to simulate different clinical scenarios, such as weak and/or strong tendon-to-bone fixation, degenerative tendon tissue, or compliant/stiff scaffolds. 

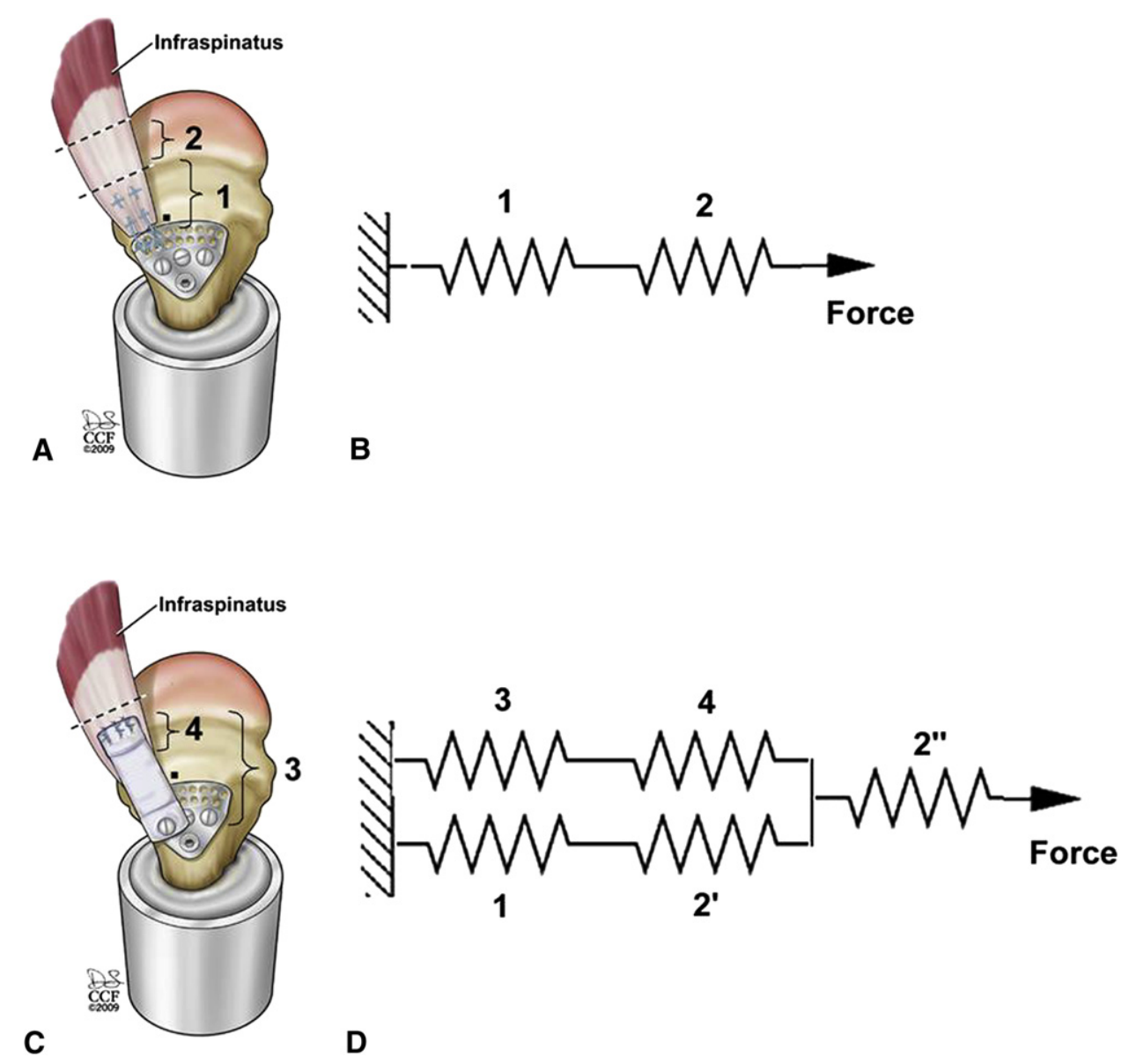

Figure 1 The non-augmented rotator cuff repairs (A) were modeled as 2 springs in series (B), namely, the bone-suture-tendon interface, ie, tendon-to-bone repair (spring \#1) and the tendon itself (spring \#2). The augmented rotator cuff repairs (C) were modeled as 5 springs in series and parallel (D), and included the bone-screw-scaffold-suture component, ie, scaffold augmentation component (spring \#3) and medial scaffold-suture-tendon interface, ie, scaffold-tendon attachment (spring \#4). Details of the surgical repair techniques and model development have previously been reported. ${ }^{2,20}$ The dotted lines represent suture markers that were placed on the tendon during experimental tests, and the black dot represents the optical marker that was placed on the bone for optical displacement measurements. Figure adapted and reprinted from Clinical Biomechanics 2010;25:751-58, Aurora A, Gatica JE, van den Bogert AJ, McCarron JA, Derwin KA. An analytical model for rotator cuff repairs. ${ }^{2}$ With permission from Elsevier.

Specifically, to simulate changes in tendon quality and/or surgical repair technique, parameter $A$ of the tendon-to-bone repair (spring \#1) or the scaffold-tendon attachment (spring \#4) was varied $\pm 50 \%$ from baseline. To simulate a change in the scaffold design, which could include changes to the scaffold mechanical properties and/or its method of fixation, parameter $A$ of the scaffold augmentation component (spring \#3) was varied $\pm 25 \%$ and $\pm 50 \%$ from baseline. Parameter $A$ is a proportionality constant associated with the load-displacement characteristics of a given spring component and does not have any particular physical significance; however, it can be varied parametrically to simulate changes in the mechanical properties of each component. It was assumed that clinically relevant variation in tendon quality and/or surgical repair technique would fall in the range of $\pm 50 \%$ of the properties of the cadaveric specimens used to develop this model.

The biomechanical performance of the repair constructs, that is, the yield load and stiffness, and the percent load carried by the scaffold augmentation component (spring \#3), were evaluated for each of the parametrically simulated model conditions. Since the model was fitted to the experimental data only up to the point of yield load (yield load was defined at the first instantaneous drop in load of at least $10 \%$ during the experimental tests), ${ }^{20}$ the maximum load predicted by the model simulations is equivalent to this yield load. The stiffness was obtained from the linear portion (between 5 and $150 \mathrm{~N}$ ) of the predicted load-displacement curves. All results are reported with respect to the nonaugmented repair condition to estimate value of using scaffold augmentation for the simulated clinical indications.

\section{Results}

\section{Parametric variation in parameter $A$}

\section{Tendon-to-bone repair (spring \#1)}

Parameter $A$ of the tendon-to-bone repair (spring \#1) was varied to simulate changes in tendon quality and/or surgical 


\begin{tabular}{|c|c|}
\hline Spring & Physical component of repair construct \\
\hline 1 & $\begin{array}{l}\text { Tendon-to-bone repair (bone-suture-tendon } \\
\text { interface) }\end{array}$ \\
\hline $2,2^{\prime}, 2^{\prime \prime}$ & $\begin{array}{l}\text { Tendon (springs } 2^{\prime} \text { and } 2^{\prime \prime} \text { are two half-springs } \\
\text { of spring 2) }\end{array}$ \\
\hline 3 & $\begin{array}{l}\text { Scaffold augmentation component (bone-screw- } \\
\text { scaffold-suture) }\end{array}$ \\
\hline 4 & $\begin{array}{l}\text { Scaffold-tendon attachment (scaffold-suture- } \\
\text { tendon interface) }\end{array}$ \\
\hline
\end{tabular}

repair technique. Results are shown in Figure 2 and summarized in Table II. The model predicted a yield load of $384 \mathrm{~N}$ and stiffness of $105 \mathrm{~N} / \mathrm{mm}$ for nonaugmented repair constructs. These are the baseline properties to which all simulated conditions are compared.

Decreasing parameter $A$ of the tendon-to-bone repair (spring \#1) by 50\% decreases the yield load (43\%) and stiffness $(62 \%)$ of nonaugmented repairs. When an augmentation scaffold is used, the same decrease in parameter $A$ resulted in only a modest decrease in yield load (4\%) and stiffness (21\%) compared with the nonaugmented baseline condition (Fig. 2, $A)$. In this scenario, the scaffold augmentation component carries approximately $45 \%$ of the total load on the construct (Fig. 2, B).

Conversely, increasing parameter $A$ of the tendon-to-bone repair (spring \#1) by $50 \%$ increases the yield load (34\%) and stiffness (38\%) of nonaugmented repairs (Fig. 2,A). When an augmentation scaffold is used, the same increase in parameter $A$ resulted in a similar increase in yield load (43\%) and stiffness (32\%) as when no scaffold is used (Fig. 2, A). In this scenario, however, the scaffold component carries approximately $25 \%$ of the total load on the construct (Fig. 2, B).

\section{Scaffold augmentation component (spring \#3)}

Parameter $A$ of the scaffold augmentation component (spring \#3) was varied to simulate a change in the scaffold design, which could include changes to the scaffold mechanical properties and/or its method of fixation. Results are shown in Figure 3 and summarized in Table II. Augmenting the repair with a prototypical polymer scaffold results in a repair construct with higher yield load (25\%) and stiffness (16\%) than the nonaugmented repair (Fig. 3, A), and the scaffold component carries approximately $31 \%$ of the total load on the construct (Fig. 3, B). Decreasing parameter $A$ of the scaffold augmentation component by $25 \%$ and $50 \%$ reduces the properties of the augmented construct to similar levels as the nonaugmented baseline repair (Fig. 3, $A$ ), and the percent total load carried by the scaffold reduces to $20 \%$ (Fig. 3, B). Increasing parameter $A$ of the scaffold augmentation component by $25 \%$ and $50 \%$ does not appreciably increase the yield load, stiffness, or load-sharing capability of the
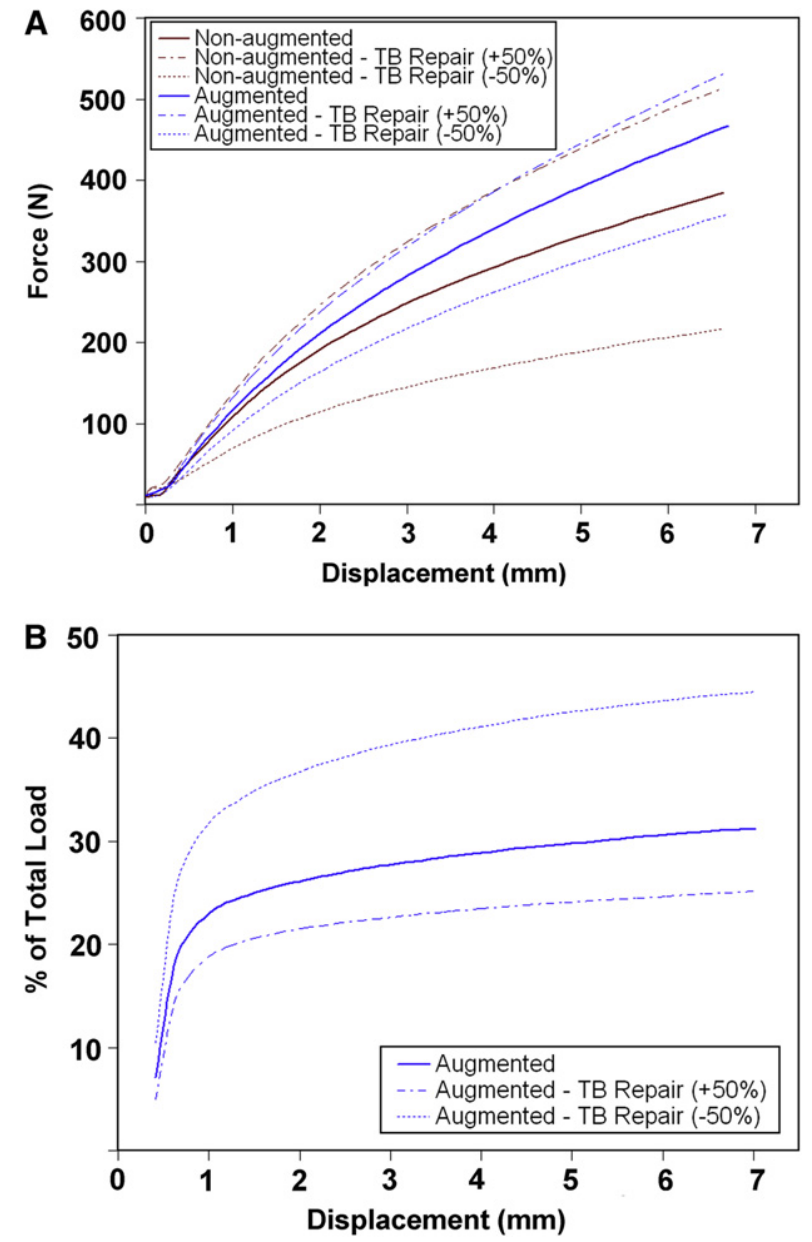

Figure 2 Parametric variation in parameter $A$ of the tendon-tobone $(T B)$ repair (spring \#1). (A) The biomechanical performance of the nonaugmented and augmented repair constructs and $(\mathbf{B})$ the percent load carried by the scaffold augmentation component for simulated tendon-to-bone repair.

augmented construct over the properties obtained with the prototypical polymer scaffold (Fig. 3, $A$ and $B$ ).

\section{Scaffold-tendon attachment (spring \#4)}

Parameter $A$ of the scaffold-tendon attachment (Spring\#4) was varied to simulate changes in tendon quality and/or scaffold attachment technique. Results are shown in Figure 4 and summarized in Table II. For repairs augmented with a prototypical polymer scaffold, decreasing parameter $A$ of the scaffold-tendon attachment by 50\% reduces the properties of the augmented repair construct to similar levels as the nonaugmented baseline repair, and the percent total load carried by the scaffold reduces to $22 \%$ (Fig. $4, A$ ). Increasing parameter $A$ of the scaffold-tendon attachment by $50 \%$ does not appreciably increase the yield load, stiffness, or loadsharing capability of the augmented construct over the properties obtained with the prototypical polymer scaffold and the baseline condition for spring \#4 (Fig. 4, B). In other words, changes to the properties of the repair construct with 
Table II Predicted biomechanical performance of repair constructs for simulated clinical scenarios*

\begin{tabular}{|c|c|c|c|c|c|}
\hline \multirow[t]{2}{*}{ Clinical scenario } & \multirow[t]{2}{*}{$\begin{array}{l}\text { Parameter } \\
A \text { variation }\end{array}$} & \multirow[t]{2}{*}{ Repair type } & \multicolumn{2}{|c|}{$\begin{array}{l}\text { Percent change from } \\
\text { nonaugmented } \\
\text { (primary repair) }\end{array}$} & \multirow{2}{*}{$\begin{array}{l}\text { Percent load } \\
\text { carried by the } \\
\text { scaffold } \\
\text { augmentation } \\
\text { component }\end{array}$} \\
\hline & & & Yield load & Stiffness & \\
\hline \multicolumn{6}{|l|}{ Tendon-to-bone repair varied (spring \#1) } \\
\hline \multirow{2}{*}{$\begin{array}{l}\text { Reduced tendon quality (ie, repair of chronic } \\
\text { degenerative tendon to bone) }\end{array}$} & \multirow[t]{2}{*}{$50 \%$ decrease } & Nonaugmented & $-43 \%$ & $-62 \%$ & $\mathrm{~N} / \mathrm{a}$ \\
\hline & & Augmented with a prototypical scaffold & $-4 \%$ & $-21 \%$ & $45 \%$ \\
\hline \multirow{2}{*}{$\begin{array}{l}\text { Improved repair strategy of tendon attachment } \\
\text { to bone }\end{array}$} & \multirow[t]{2}{*}{$50 \%$ increase } & Nonaugmented & $+34 \%$ & $+38 \%$ & N/A \\
\hline & & Augmented with a prototypical scaffold & $+43 \%$ & $+32 \%$ & $25 \%$ \\
\hline \multicolumn{6}{|l|}{ Scaffold augmentation component varied (spring \#3) } \\
\hline \multirow{5}{*}{$\begin{array}{l}\text { Change in scaffold mechanical properties and/ } \\
\text { or its method of fixation }\end{array}$} & Prototypical polymer & Augmented & $+25 \%$ & $+16 \%$ & $31 \%$ \\
\hline & $25 \%$ decrease & & $+20 \%$ & $+4 \%$ & $28 \%$ \\
\hline & $50 \%$ decrease & & $+12 \%$ & no change & $20 \%$ \\
\hline & $25 \%$ increase & & $+29 \%$ & $+18 \%$ & $34 \%$ \\
\hline & $50 \%$ increase & & $+32 \%$ & $+20 \%$ & $36 \%$ \\
\hline \multicolumn{6}{|l|}{ Scaffold-tendon attachment varied (spring \#4) } \\
\hline $\begin{array}{l}\text { Reduced tendon quality and/or reduced repair } \\
\text { strategy of scaffold attachment to tendon }\end{array}$ & $50 \%$ decrease & Augmented with a prototypical scaffold & $+14 \%$ & No change & $22 \%$ \\
\hline $\begin{array}{l}\text { Improved repair strategy of scaffold } \\
\text { attachment to tendon }\end{array}$ & $50 \%$ increase & & $+31 \%$ & $+19 \%$ & $36 \%$ \\
\hline
\end{tabular}

$N / A$, not applicable.

* All results are reported with respect to the non-augmented repair condition (yield load: $384 \mathrm{~N}$, stiffness: $105 \mathrm{~N} / \mathrm{mm}$ ), in order to estimate value of scaffold augmentation for the simulated clinical scenarios. 

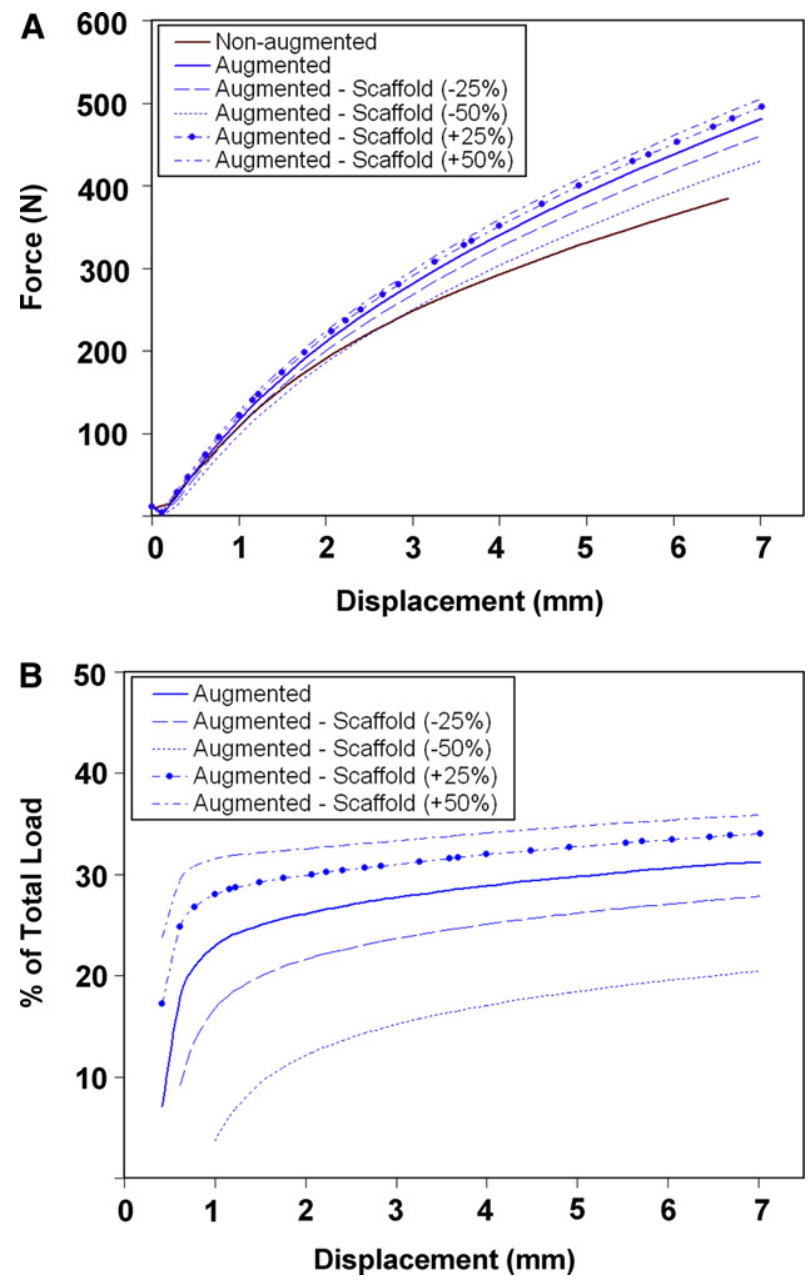

Figure 3 Parametric variation in parameter $A$ of the scaffold augmentation component (spring \#3). (A) The biomechanical performance of the augmented repair construct and (B) the percent load carried by the scaffold augmentation component for simulated scaffold augmentation component.

variation in the scaffold-tendon attachment properties were essentially the same as when the properties of the scaffold augmentation component (spring \#3) were varied to the same degree (Fig. 4, $A$ and $B$ ).

\section{Discussion}

Scaffold augmentation may be a viable strategy to improve the initial biomechanical properties of a rotator cuff repair construct and thereby reduce the incidence of repair failure. However, numerous questions remain about the appropriate scaffold properties, surgical application techniques, and load-sharing abilities of a scaffold when used in a rotator cuff repair construct. To investigate these questions and enhance our understanding of the basic mechanics of scaffold augmentation, we recently developed and validated a spring-network model for nonaugmented and augmented
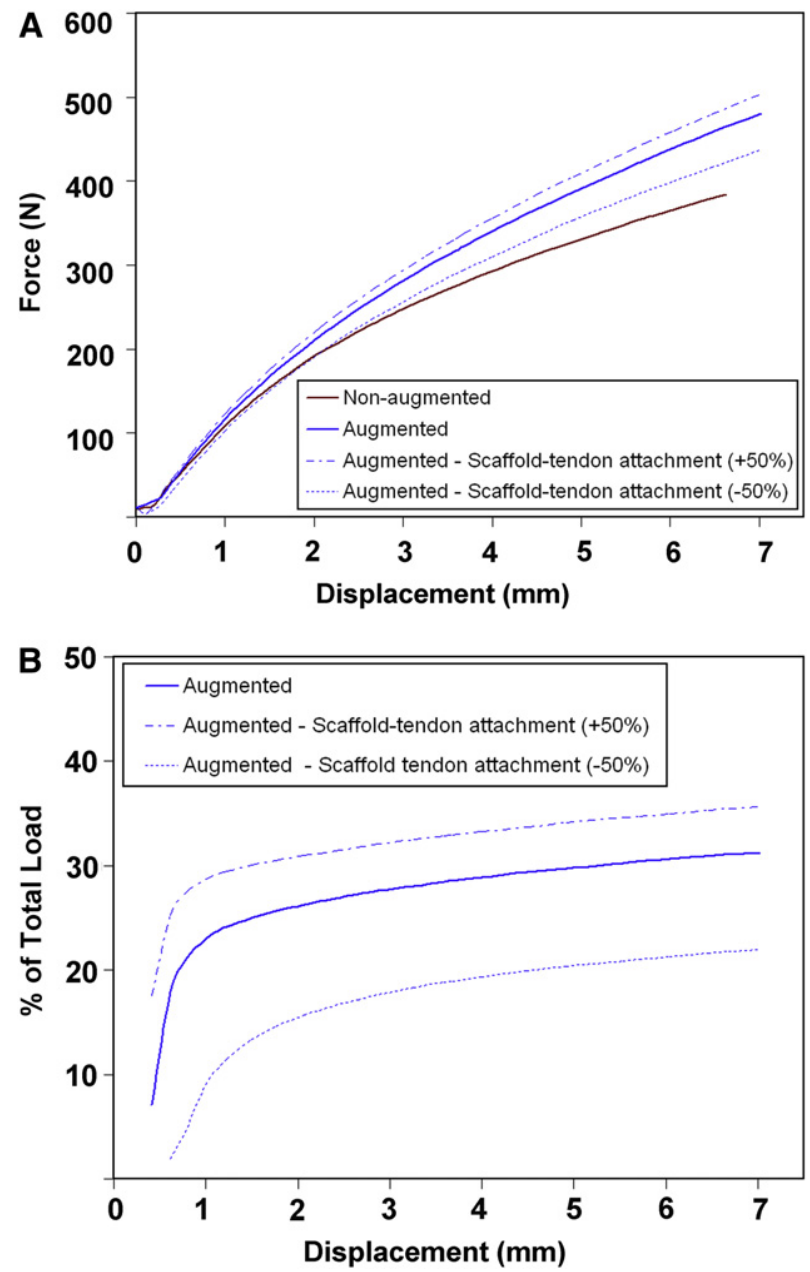

Figure 4 Parametric variation in parameter $A$ of the scaffoldtendon attachment (spring 4). (A) The biomechanical performance of the augmented repair construct and (B) the percent load carried by the scaffold augmentation component for simulated scaffold-tendon attachment.

human rotator cuff repairs. ${ }^{2}$ The objectives of the current study were to use this model to predict (1) the manner in which simulated changes to components of the tendon repair, such as reduced tendon quality, altered surgical technique, and different scaffold designs, influence the biomechanical performance (yield load and stiffness) of the repair construct, and (2) the percent load carried by the scaffold augmentation component of the repair construct in each of these simulated clinical scenarios.

The model was developed and validated from our in vitro experimental study of nonaugmented and augmented human rotator cuff repairs, performed using a polymer scaffold designed to have stiffness and ultimate load comparable with human rotator cuff tendon. ${ }^{20}$ Except for a small portion of the data at large displacement values, the experimental data fell within the 95\% confidence interval of the model, thus validating the model as a predictive tool for investigating the basic mechanics of scaffold augmentation. ${ }^{2}$ 
The model predicts that augmenting a tendon repair with a polymer scaffold designed to have tendon-like mechanical properties results in a repair construct with modestly higher yield load (25\%) and stiffness (16\%) than the nonaugmented repair condition. The model also predicts that the scaffold component of the repair construct carries $31 \%$ of the total load on the repair. The model predicts only slight further increases in repair construct stiffness or yield load when the mechanical properties of the scaffold augmentation component and/or its attachment to tendon are increased. Decreasing the properties of the scaffold augmentation component itself and/or its attachment to the repaired tendon reduces the properties of the of the overall augmented repair construct to similar levels as the nonaugmented repair. Together, these findings suggest that to provide modest improvements to the stiffness and yield load of nonaugmented repairs in healthy tendon tissue, the scaffold must have mechanical properties similar to that of tendon tissue. However, the results also suggest that applying a scaffold with supraphysiologic stiffness will not translate into yet stiffer or stronger repairs.

Importantly, the model predicts that in the presence or absence of an augmentation scaffold, the mechanical properties of the overall repair construct are most influenced by the properties of the primary tendon-to-bone repair. The model predicts that decreasing the properties of the tendon-to-bone repair (ie, repair of a chronic degenerative tendon, fixation in osteopenic bone, or a poorly performed surgical repair technique) will appreciably decrease the yield load (43\%) and stiffness (62\%) of the construct. The model predicts that scaffold augmentation in this setting can largely mitigate this drop in properties and that the scaffold will carry approximately $45 \%$ of the total load on the repair construct. This result suggests that scaffold augmentation would be particularly advantageous when repairing poor-quality tendon.

Conversely, the model also predicts that increasing the properties of the tendon-to-bone repair (perhaps representing an improved tendon-to-bone repair strategy), will appreciably increase the yield load (34\%) and stiffness (38\%) of the repair construct even without scaffold augmentation. In this case, scaffold augmentation provides minimal further improvement in construct properties, although $25 \%$ of the total load on the overall construct would still be carried by the scaffold component of the repair.

It is important to note that because the mechanical properties of the primary tendon-to-bone repair most influence the overall mechanical performance of the repair construct, using a surgical repair technique that maximizes the strength and stability of the direct tendon-to-bone fixation site is essential, even if repair augmentation with a scaffold is anticipated. Surgical repair strategies that compromise the fixation strength at the tendon-to-bone repair site in favor of improved scaffold fixation are unlikely to confer mechanical benefit to the overall repair construct.
Several limitations should be noted in interpreting the findings of the study: first, the objectives of the study were met by parametrically simulating a simplified rotator cuff repair model that was validated for one surgical repair technique, one type of scaffold, and tested under one loading condition. ${ }^{2,20}$ Hence, the results reported are dependent on the particular experimental conditions tested.

Second, the parameter $A$ does not have any direct physical corollary. It is a proportionality constant associated with the load-displacement characteristics of a given spring component. Although varying parameter $A$ allowed us to simulate the model for clinical scenarios that may be representative of change in tendon quality, altered surgical techniques, and/or scaffold designs, the absolute translation of our model predictions to clinical practice must be done judiciously.

Third, the model does not account for the biologic processes of healing and remodeling. Hence, the results of the study are only applicable to the immediate postoperative period.

Fourth, we modeled failure loading after cyclic preconditioning and not the more physiological cyclic loading condition.

Despite these limitations, we believe the model is useful to enhance our understanding of the basic mechanics of scaffold augmentation. Future work will develop and apply the spring-network model to a more clinically relevant repair and loading conditions in a human cadaver model: full-thickness supraspinatus tendon repairs (with and without scaffold augmentation) exposed to a cyclic loading protocol.

\section{Conclusion}

A previously validated human rotator cuff repair model was used to simulate changes in tendon quality, altered surgical technique(s) and different scaffold designs. This model allows predictions of the biomechanical performance of nonaugmented and augmented repair constructs as well as the percent load carried by the scaffold augmentation component for various clinically relevant scenarios. The model predicts that the yield load and stiffness of a rotator cuff repair at the time of surgery may be modestly increased by augmenting the repair with a scaffold, which has tendon-like properties. However, the model also suggests that engineering a scaffold with supraphysiologic stiffness may not translate into yet stiffer or stronger repairs.

Importantly, the model also predicts that the mechanical properties of a repair construct are most influenced by the properties of the tendon-to-bone repair. This result illustrates the need to prioritize the primary tendon-to-bone repair site fixation, even if repair augmentation with a scaffold is anticipated. In the clinical setting of a weak tendon-to-bone repair, scaffold augmentation will significantly off-load the 
repair and largely mitigate the poor construct properties, based on the current model predictions.

To our knowledge, this work provides for the first time, information about the load-sharing ability of augmentation scaffolds used for rotator cuff repair, and offers unique insight into how changes to various components of the repair may influence the biomechanical performance of the repair construct. Given the increasing prevalence of scaffold devices being developed and marketed for rotator cuff repair, the information provided by this study is of great clinical relevance as surgeons endeavor to further understand the role of scaffolds for rotator cuff repair augmentation.

Importantly, the model simulations may be used to direct and inform the design of new repair strategies aimed at improving the biomechanical performance of rotator cuff repairs and may have broader implications for understanding the basic mechanics of scaffold augmentation of other soft tissue repairs as well. The simulations suggest that future efforts in the field of rotator cuff repair augmentation may be directed toward strategies that strengthen the tendon-to-bone repair or toward engineering scaffolds with tendon-like mechanical properties that also promote rapid or effective biologic healing. Future work will develop and apply the model to a more clinically relevant rotator cuff repair and loading conditions.

\section{Reference}

1. Accousti KJ, Flatow EL. Technical pearls on how to maximize healing of the rotator cuff. Instr Course Lect 2007;56:3-12. ISSN: 0065-6895.

2. Aurora A, Gatica JE, den Bogert AJ, McCarron JA, Derwin KA. An analytical model for rotator cuff repairs. Clin Biomechanics 2010;25: 751-8. doi:10.1016/j.clinbiomech.2010.05.010

3. Aurora A, McCarron J, Iannotti JP, Derwin K. Commercially available extracellular matrix materials for rotator cuff repairs: state of the art and future trends. J Shoulder Elbow Surg 2007;16(5 suppl):S171-8. doi:10.1016/j.jse.2007.03.008
4. Barber FA, Herbert MA, Boothby MH. Ultimate tensile failure loads of a human dermal allograft rotator cuff augmentation. Arthroscopy 2008;24:20-4. doi:10.1016/j.arthro.2007.07.013

5. Bartolozzi A, Andreychik D, Ahmad S. Determinants of outcome in the treatment of rotator cuff disease. Clin Orthop Rel Res 1994;308:90-7.

6. Bishop J, Klepps S, Lo IK, Bird J, Gladstone JN, Flatow EL. Cuff integrity after arthroscopic versus open rotator cuff repair: a prospective study. J Shoulder Elbow Surg 2006;15:290-9. doi:10.1016/j.jse. 2005.09.017

7. Boileau P, Brassart N, Watkinson DJ, Carles M, Hatzidakis AM, Krishnan SG. Arthroscopic repair of full-thickness tears of the supraspinatus: does the tendon really heal? J Bone Joint Surg Am 2005;87:1229-40. doi:10.2106/JBJS.D.02035

8. Cofield RH, Parvizi J, Hoffmeyer PJ, Lanzer WL, Ilstrup DM, Rowland CM. Surgical repair of chronic rotator cuff tears. A prospective long-term study. J Bone Joint Surg Am 2001;83-A:71-7.

9. Coons DA, Alan Barber F. Tendon graft substitutes-rotator cuff patches. Sports Med Arthrosc 2006;14:185-90.

10. Derwin KA, Badylak SF, Steinmann SP, Iannotti JP. Extracellular matrix scaffold devices for rotator cuff repair. J Shoulder Elbow Surg 2010;19:467-76. doi:10.1016/j.jse.2009.10.020

11. Franceschi F, Ruzzini L, Longo UG, Martina FM, Zobel BB, Maffulli N, et al. Equivalent clinical results of arthroscopic single-row and double-row suture anchor repair for rotator cuff tears: a randomized controlled trial. Am J Sports Med 2007;35:1254-60. doi:10.1177/ 0363546507302218

12. Galatz LM, Ball CM, Teefey SA, Middleton WD, Yamaguchi K. The outcome and repair integrity of completely arthroscopically repaired large and massive rotator cuff tears. J Bone Joint Surg Am 2004;86-A:219-24.

13. Gazielly DF, Gleyze P, Montagnon C. Functional and anatomical results after rotator cuff repair. Clin Orthop Rel Res 1994;304:43-53.

14. Gerber C, Fuchs B, Hodler J. The results of repair of massive tears of the rotator cuff. J Bone Joint Surg Am 2000;82:505-15.

15. Goutallier D, Postel JM, Gleyze P, Leguilloux P, Van Driessche S. Influence of cuff muscle fatty degeneration on anatomic and functional outcomes after simple suture of full-thickness tears. J Shoulder Elbow Surg 2003;12:550-4. doi:10.1016/S1058-2746(03)00211-8

16. Harryman DT, Mack LA, Wang KY, Jackins SE, Richardson ML, Matsen FA III. Repairs of the rotator cuff. Correlation of functional results with integrity of the cuff. J Bone Joint Surg Am 1991;73-A:982-9.

17. Ide J, Maeda S, Takagi K. A comparison of arthroscopic and open rotator cuff repair. Arthroscopy 2005;21:1090-8. doi:10.1016/j.arthro. 2005.05.010

18. Klintberg IH, Gunnarsson AC, Svantesson U, Styf J, Karlsson J. Early loading in physiotherapy treatment after full-thickness rotator cuff repair: a prospective randomized pilot-study with a two-year followup. Clin Rehabil 2009;23:622-38. doi:10.1177/0269215509102952

19. Koo SS, Burkhart SS. Rehabilitation following arthroscopic rotator cuff repair. Clin Sports Med 2010;29:203-11. doi:10.1016/j.csm.2009.12.001

20. McCarron JA, Milks RA, Chen X, Iannotti JP, Derwin KA. Improved time-zero biomechanical properties using poly-L-lactic acid graft augmentation in a cadaveric rotator cuff repair model. J Shoulder Elbow Surg 2010;19:688-96. doi:10.1016/j.jse.2009.12.008

21. Saridakis P, Jones G. Outcomes of single-row and double-row arthroscopic rotator cuff repair: a systematic review. J Bone Joint Surg Am 2010;92:732-42. doi:10.2106/JBJS.I.01295

22. Thomopoulos S, Soslowsky LJ, Flanagan CL, Tun S, Keefer CC, Mastaw J, et al. The effect of fibrin clot on healing rat supraspinatus tendon defects. J Shoulder Elbow Surg 2002;11:239-47. doi:10.1067/ mse. 2002.122228

23. Uhthoff HK, Matsumoto F, Trudel G, Himori K. Early reattachment does not reverse atrophy and fat accumulation of the supraspinatus-an experimental study in rabbits. J Orthop Res 2003;21:386-92. doi:10. 1016/S0736-0266(02)00208-5

24. Vitale MA, Vitale MG, Zivin JG, Braman JP, Bigliani LU, Flatow EL. Rotator cuff repair: an analysis of utility scores and cost-effectiveness. J Shoulder Elbow Surg 2007;16:181-7. doi:10.1016/j.jse.2006.06.013 